\title{
Mapping Co-regulatory Interactions among Ligand Binding sites in RyR1
}

\author{
Venkat Chirasani $^{1}$, Konstantin Popov ${ }^{1}$, Gerhard Meissner ${ }^{1}$, and Nikolay Dokholyan ${ }^{2}$ \\ ${ }^{1}$ University of North Carolina at Chapel Hill School of Medicine \\ ${ }^{2}$ Penn State College of Medicine Department of Pharmacology
}

May 3, 2021

\begin{abstract}
Ryanodine receptor 1 (RyR1) is an intracellular calcium ion $(\mathrm{Ca} 2+)$ release channel required for skeletal muscle contraction. Although cryo-electron microscopy identified binding sites of three coactivators Ca2+, ATP and caffeine (CFF), the mechanism of co-regulation and synergy of these activators is unknown. Here, we report allosteric connections among the three ligand binding sites and pore region in (i) Ca2+ bound-closed, (ii) ATP/CFF bound- closed, (iii) Ca2+/ATP/CFF bound-closed, and (iv) $\mathrm{Ca} 2+/ \mathrm{ATP} / \mathrm{CFF}$ bound-open RyR1 states. We identified two dominant interactions that mediate interactions between the $\mathrm{Ca} 2+$ binding site and pore region in $\mathrm{Ca} 2+$ bound-closed state, which partially overlapped with the pore communications in ATP/CFF bound-closed RyR1 state. In Ca2+/ATP/CFF bound-closed and -open RyR1 states, co-regulatory interactions were analogous to communications in the $\mathrm{Ca} 2+$ bound-closed and ATP/CFF bound- closed states. Both ATP- and CFF- binding sites mediate communication between the Ca2+ binding site and the pore region in Ca2+/ATP/CFF bound - open RyR1 structure. We conclude that $\mathrm{Ca} 2+, \mathrm{ATP}$, and $\mathrm{CFF}$ propagate their effects to the pore region through a network of overlapping interactions that mediate allosteric control and molecular synergy in channel regulation.
\end{abstract}

\section{Hosted file}

cpmd.Proteins21.pdf available at https://authorea.com/users/411657/articles/520620-mappingco-regulatory-interactions-among-ligand-binding-sites-in-ryr 1 